\title{
Perfil de morbidade e hospitalização entre adolescentes da região de Ribeirão Preto/SP
}

\author{
Morbidity profile and hospitalization of adolescents at \\ Ribeirão Preto $(S P)$ region
}

\author{
Luiz Antonio Del Ciampo, leda Regina Lopes Del Ciampo
}

\begin{abstract}
RESUMO
Modelo de estudo: levantamento epidemiológico. Introdução: A adolescência é uma fase de grandes transformações biológicas e emocionais associadas à definição do papel social, tornando-se importante o conhecimento dos problemas de saúde mais prevalentes nessa faixa etária a fim de melhor direcionar ações e recursos visando à assistência e aos programas preventivos.

Objetivos: conhecer o perfil de morbidade hospitalar entre adolescentes da região de Ribeirão Preto (SP) e sua evolução temporal nos anos de 1988, 1997 e 2006.

Métodos: levantamento de dados do Centro de Processamento de Dados Hospitalares do Departamento de Medicina Social da Faculdade de Medicina de Ribeirão Preto da Universidade de São Paulo, de todas as internações de adolescentes nos 34 hospitais dos 26 municípios da Divisão Regional de Saúde de Ribeirão Preto (SP), nos anos de 1988, 1997 e 2006.

Resultados: os principais diagnósticos de internação na faixa etária de 10 a 14 anos foram pneumonias (primeira causa de hospitalização em 1988 e 1997), diarréias e apendicite em todos os anos, enquanto as intoxicações e as neoplasias registradas apenas no ano de 1988. Para os adolescentes com mais de 15 anos foram elevadas as taxas de internação devido aos problemas relacionados à gravidez, parto e puerpério, embora pneumonias, diarréias e apendicite ainda sejam diagnósticos importantes. As internações hospitalares e os óbitos predominaram no sexo masculino, em todos os anos. O sexo masculino sempre apresentou maiores taxas de mortalidade que o feminino e as proporções dos óbitos por internações indicaram freqüências mais elevadas entre os adolescentes de 10 a 14 anos. Conclusões: o conhecimento do perfil de morbidade entre os adolescentes propicia a adoção de importantes ações exeqüíveis em nível primário que devem ser incorporadas ao programas de Puericultura e de Hebiatria e nos ambulatórios de Pré-Natal.
\end{abstract}

Palavras-chave: Adolescente. Hospitalização. Morbidade. Mortalidade.

${ }^{1}$ Docentes, Departamento de Puericultura e Pediatria da Faculdade de Medicina de Ribeirão Preto da Universidade de São Paulo
Correspondência: Luiz Antonio Del Ciampo Departamento de Puericultura e Pediatria Faculdade de Medicina de Ribeirão Preto - USP Avenida Bandeirantes, 3900 14049-900 - Ribeirão Preto/SP delciamp@fmrp.usp.br

Artigo recebido em 27/09/2010 Aprovado para publicação em 05/06/2011 


\section{Introdução}

A adolescência pode ser compreendida como uma fase da vida em que os indivíduos experimentam importantes e rápidas transformações biológicas, fisiológicas e emocionais associadas à definição de seu papel social. Esse conjunto de modificações que ocorrem durante a segunda década da vida predispõe o adolescente à maior vulnerabilidade devido aos riscos para sua saúde e qualidade de vida, principalmente relacionados a comportamentos associados ao tabagismo, consumo de álcool e drogas, relações sexuais desprotegidas, dietas inadequadas e sedentarismo que compõem o cenário das causas diretamente relacionadas à morbidade e mortalidade entre os adolescentes. ${ }^{1,2,3}$

Atualmente, cerca de $20 \%$ da população brasileira situa-se na faixa etária dos dez aos dezenove anos, o que compreende mais de 38 milhões de adolescentes $(47,8 \%$ com idades entre 10 e 14 anos e $52,2 \%$ na faixa dos 15 aos 19 anos), ${ }^{4}$ o que vale dizer que, em termos populacionais, corresponderia ao $34^{\circ}$ pais do mundo, caso contasse apenas com seus adolescentes, o que destaca a importância de se desenvolverem estudos que ampliem o conhecimento sobre esse segmento da população. Por outro lado, torna-se também importante o conhecimento sobre o impacto econômico que os problemas de saúde mais prevalentes na população acarretam à sociedade, em função da aplicação dos recursos poderem ser direcionados adequadamente à assistência e aos programas preventivos. ${ }^{5}$

Ao se tentar compreender o adolescente como paciente merece ser destacado que a hospitalização, decorrente do tratamento de doenças crônicas ou procedimentos cirúrgicos, marca a adolescência física e emocionalmente, alterando a auto-imagem e comprometendo a auto-estima ao determinar o afastamento do convívio familiar e social. ${ }^{6,7}$ Portanto, identificar os problemas de saúde mais prevalentes pode oferecer subsídios para os serviços de atendimento e os pro- gramas de prevenção, contribuindo para elaboração de políticas públicas e efetivação de medidas preventivas, de controle e de redução das principais causas de morbidade e de mortalidade nessa faixa etária.

Este trabalho tem por objetivo conhecer o perfil de morbidade hospitalar entre adolescentes da região de Ribeirão Preto (SP) e sua evolução temporal, nos anos de 1988, 1997 e 2006.

\section{Métodos}

Trata-se de um levantamento de dados obtidos dos registros do Centro de Processamento de Dados Hospitalares (CPDH) do Departamento de Medicina Social da Faculdade de Medicina de Ribeirão Preto da Universidade de São Paulo, que centraliza os dados de internações hospitalares de todos os hospitais do município. Este Centro é abastecido com informações mediante o recebimento de documentos padronizados, desde 1970, sobre a assistência médico-hospitalar na cidade de Ribeirão Preto. Para o presente estudo, analisarma-se todas as internações de pacientes na faixa etária dos dez aos dezenove anos, nos 34 hospitais dos 26 municípios que integram a Divisão Regional de Saúde - DIR XIII, de Ribeirão Preto (SP), nos anos de 1988, 1997 e 2006. Para cada ano os dados pesquisados foram o número total de internações distribuído por sexo e faixas etárias (10 a 14 anos e 15 a 19 anos), o número de óbitos e a relação dos principais diagnósticos de internação hospitalar.

\section{Resultados}

A Tabela 1 apresenta a distribuição dos adolescentes internados nos hospitais da região de Ribeirão Preto (SP) nos anos de 1988, 1997 e 2006, segundo a faixa etária e o sexo. Pode-se observar que, para os adolescentes com idades entre 10 e 14 anos, as frequiências de internação para o sexo masculino sempre

\section{Tabela 1}

Distribuição dos adolescentes internados nos hospitais da região de Ribeirão Preto (SP) segundo sexo e faixa etária nos anos de 1988, 1997 e 2006.

\begin{tabular}{lrrrrrrr}
\hline \multirow{2}{*}{ sexo } & idade(anos) & \multicolumn{2}{c}{1988} & \multicolumn{2}{c}{1997} & \multicolumn{2}{c}{2006} \\
\cline { 2 - 8 } masculino & $10-14$ & 2046 & 16,6 & 2619 & 16,8 & 2097 & 16,9 \\
& $15-19$ & 2486 & 20,1 & 2884 & 18,5 & 2397 & 19,3 \\
\multirow{2}{*}{ feminino } & $10-14$ & 1538 & 12,4 & 2080 & 13,3 & 1763 & 14,2 \\
& $15-19$ & 6252 & 50,7 & 8010 & 51,3 & 6148 & 49,5 \\
\hline \multirow{2}{*}{ Total } & & 12322 & 100 & 15593 & 100 & 12405 & 100 \\
\hline
\end{tabular}


foram superiores às do feminino, situação que se inverte quando considerada a faixa etária que vai dos 15 aos 19 anos.

Na Tabela 2 estão apresentadas as cinco principais causas de internação de adolescentes de adolescentes de 10 a 14 anos nos anos de 1988, 1997 e 2006. Nela se destacam as pneumonias (primeira causa de hospitalização em 1988 e 1997), diarréias e apendicite que ocorreram em todos os anos, enquanto as intoxicações e as neoplasias aparecem apenas no ano de 1988.

O perfil de morbidade apresentado pela Tabela 3 destaca as elevadas proporções de internação devido aos problemas relacionados à gravidez, parto e puerpério. Excluída essa primeira causa de interna- ção em todos os anos, ao se observar o perfil que inclui ambos os sexos, pode-se identificar que ainda as pneumonias, as diarréias e apendicite são diagnósticos importantes entre os adolescentes na faixa etária dos 15 aos 19 anos.

Na Tabela 4 estão descritos o percentual de internações hospitalares e de óbitos, segundo o sexo e a faixa etária dos adolescentes. Houve predomínio do sexo feminino nas internações para a faixa etária de 15 a 19 anos, enquanto que o sexo masculino contribuiu mais para as internações na faixa etária de 10 a 14 anos e nos óbitos, em ambas as faixas. A distribuição das proporções dos óbitos por internações, apresentada na Tabela 5, destaca as maiores freqüências entre os adolescentes de 10 a 14 anos.

\section{Tabela 2}

Distribuição de frequiências das cinco principais causas de internação hospitalar de adolescentes (10-14 anos) na região de Ribeirão Preto (SP) nos anos de 1988, 1997 e 2006

\begin{tabular}{|c|c|c|c|c|c|}
\hline \multicolumn{2}{|c|}{1988} & \multicolumn{2}{|c|}{1997} & \multicolumn{2}{|c|}{2006} \\
\hline Causa & $\%$ & Causa & $\%$ & Causa & $\%$ \\
\hline Pneumonia & 6,1 & Pneumonia & 4,8 & Apendicite & 5,5 \\
\hline Diarréia & 4,2 & Apendicite & 4,5 & Fratura & 5,1 \\
\hline Neoplasia & 3,1 & Acidente & 3,7 & Pneumonia & 4,0 \\
\hline Intoxicação & 2,9 & Diarréia & 3,5 & Diarréia & 3,4 \\
\hline Apendicite & 2,8 & Adenoamigdalectomia & 3,2 & Adenoamigdalectomia & 3,3 \\
\hline
\end{tabular}

\section{Tabela 3}

Distribuição de freqüências das cinco principais causas de internação hospitalar de adolescentes (15 -19 anos) na região de Ribeirão Preto (SP) nos anos de 1988, 1997 e 2006

\begin{tabular}{rrrrrr}
\hline \multicolumn{2}{c}{1988} & \multicolumn{2}{c}{$\mathbf{1 9 9 7}$} & \multicolumn{2}{c}{$\mathbf{2 0 0 6}$} \\
\hline Causa & $\%$ & Causa & $\%$ & Causa & $\%$ \\
\hline Gravidez, parto e puerpério & 38,4 & Gravidez, parto e puerpério & 44,6 & Gravidez, parto e puerpério & 33,1 \\
Pneumonia & 3,7 & Pneumonia & 2,1 & Apendicite & 2,7 \\
Diarréia & 1,9 & Apendicite & 2,1 & Sinusite & 1,6 \\
Intoxicação & 1,5 & Diarréia & 1,3 & Pneumonia & 1,5 \\
Apendicite & 1,4 & Intoxicação & 1,1 & Diarréia & 1,3 \\
\hline
\end{tabular}

\section{Tabela 4}

Distribuição percentual das internações hospitalares e dos óbitos de adolescentes nos hospitais da região de Ribeirão Preto (SP) segundo faixa etária e sexo nos anos de 1988, 1997 e 2006.

\begin{tabular}{lrrrrrrrrrrrr}
\hline & \multicolumn{4}{c}{1988} & \multicolumn{4}{c}{$\mathbf{1 9 9 7}$} & \multicolumn{3}{c}{$\mathbf{2 0 0 6}$} \\
\cline { 2 - 13 } & \multicolumn{2}{c}{ masc } & \multicolumn{2}{c}{ fem } & \multicolumn{2}{c}{ masc } & fem & \multicolumn{1}{c}{ masc } & \multicolumn{1}{c}{ fem } \\
\hline Idade em anos & $10-14$ & $15-19$ & $10-14$ & $15-19$ & $10-14$ & $15-19$ & $10-14$ & $15-19$ & $10-14$ & $15-19$ & $10-14$ & $15-19$ \\
Internação hospitalar & 57,0 & 28,4 & 43,0 & 71,6 & 55,7 & 26,5 & 44,3 & 73,5 & 54,3 & 28,0 & 45,7 & 72,0 \\
Óbito & 7,3 & 8,8 & 4,5 & 3,3 & 8,7 & 14,2 & 4,3 & 3,3 & 6,7 & 12,5 & 5,6 & 2,7 \\
\hline
\end{tabular}




\section{Tabela 5}

Distribuição da proporção de óbitos por internações de adolescentes internados nos hospitais da Região de Ribeirão Preto (SP) nos anos de 1988, 1997 e 2006.

\begin{tabular}{lcc}
\hline & $\mathbf{1 0} \mathbf{- 1 4}$ anos & $\mathbf{1 5} \boldsymbol{- 1 9}$ anos \\
\hline 1988 & 6,13 & 4,92 \\
1997 & 6,80 & 6,24 \\
2006 & 6,21 & 5,50 \\
\hline
\end{tabular}

\section{Discussão}

A adolescência é considerada uma fase da vida de menor vulnerabilidade quando comparada à infância e apresenta taxas de mortalidade inferiores àquelas observadas em outras faixas etárias. Por sua vez, o perfil de morbidade hospitalar e de mortalidade dos adolescentes evidencia como principais agravos as denominadas "causas evitáveis", principalmente relacionadas ao trânsito, violência, abuso de substâncias e à gravidez. ${ }^{3}$ No Brasil, enquanto que no final da década de 1970, traumas e violência causaram mais de $45 \%$ das mortes de adolescentes (atingindo 95,5/100.000 na faixa etária dos 15 aos 19 anos), no ano 2000 essas causas foram associadas a mais de $60 \%$ dos óbitos, com destaque para os acidentes de trânsito. ${ }^{8,9}$

Os resultados encontrados por este estudo apontam que, para a faixa etária de 10 a 14 anos, as frequiências de internações hospitalares sempre foram maiores para o sexo masculino que para o feminino. $\mathrm{O}$ inverso foi observado para os adolescentes de $15 \mathrm{a}$ 19 anos, em que houve predomínio do sexo feminino nas internações. Quanto aos óbitos houve predomínio no sexo masculino em todos os anos e para as duas faixas etárias. Estes achados estão de acordo com resultados obtidos por outros autores. Em Londrina (PR) estudo realizado com adolescentes atendidos em um hospital público encontrou $54,1 \%$ pacientes do sexo feminino, sendo a morbidade predominante devido às doenças infecciosas e parasitárias $(26,5 \%)$, enquanto que para o sexo masculino os principais diagnósticos foram lesões e envenenamentos e algumas outras consequiências de causas externas $(30,5 \%) .{ }^{10}$

Entre adolescentes da cidade de Mogi das Cruzes (SP), no período de 2000 a 2004, foi encontrado coeficiente de mortalidade mais elevado no sexo masculino, que teve como principais causas de morte as causas externas $(42,8 \%)$, doenças do aparelho res- piratório $(2,4 \%)$ e neoplasias $(1,2 \%)$, enquanto que o sexo feminino foi mais acometido pelas causas externas $(12,9 \%)$, doenças do aparelho circulatório $(2,5 \%)$ e doenças do sistema nervoso $(1,8 \%) .{ }^{11} \mathrm{Em}$ Botucatu (SP), no período de 1984 a 1993 a mortalidade foi maior na faixa etária dos 15 aos 19 anos $(63,7 \%)$ e no sexo masculino. Os principais diagnósticos foram causas externas, neoplasias e doenças do aparelho circulatório. Dentre as causas externas sobressaíram os acidentes de trânsito, com armas de fogo e afogamento. ${ }^{12}$

O perfil de morbidade encontrado entre os adolescentes de 10 a 14 anos hospitalizados na região de Ribeirão Preto destaca as pneumonias (primeira causa de hospitalização em 1988 e 1997), diarréias e apendicite em todos os anos, enquanto as intoxicações e as neoplasias apareceram apenas no ano de 1988. Quando se analisa o grupo de adolescentes com 15 anos ou mais, nota-se que o perfil de morbidade apresenta elevadas proporções de internação devido aos problemas relacionados à gravidez, parto e puerpério. Além dessa primeira causa de internação aparecer em todos os anos, pode-se identificar que ainda pneumonias, diarréias e apendicite são diagnósticos importantes entre os adolescentes na faixa etária dos 15 aos 19 anos.

Estudo realizado na Sérvia apontou que as causas mais frequentes de hospitalização entre adolescentes foram doenças respiratórias, doenças do aparelho digestivo e acidentes e outras causas externas. Foi observado também que as taxas de mortalidade por 100.000 adolescentes aumentaram de 11,65 em 1988 para 16,18 em 2004, sendo que a principal causa de mortalidade para o sexo masculino foram os tumores enquanto as causas externas foram mais prevalentes no sexo feminino. ${ }^{13}$

Dados registrados no Sistema Nacional de Informações Tóxico-Farmacológicas (SINITOX), no período de 1999 a 2001, mostram que os adolescentes responderam por $15,5 \%$ de todos os casos notificados e por $10,8 \%$ dos óbitos, o que representa a terceira faixa etária mais atingida com relação aos registros de casos e a quinta quanto aos óbitos. Para os adolescentes de 10 a 14 anos, as principais causas foram venenos animais $(33,7 \%)$ e medicamentos $(25,7 \%)$, enquanto que para a faixa etária de 15 a 19 anos foram medicamentos (33\%) e venenos animais $(21,4 \%){ }^{14}$

Nascimento et al 2003, em estudo realizado para descrever os custos dos principais grupos de causas e 
causas específicas de internações de adolescentes nos hospitais da rede integrada do SUS em Salvador (BA), encontraram que as internações foram relacionadas à gravidez, parto e puerpério $(54,9 \%$, sendo $85 \%$ dos casos na faixa etária de 15 a 19 anos), lesões, envenenamentos e outras consequências de causas externas $(9,5 \%)$ e doenças do aparelho digestivo $(6,0 \%) .{ }^{15}$ Já na cidade de Maringá (PR), a principal causa de internação hospitalar entre as meninas foi relacionada à gravidez, parto e puerpério e, entre os homens, as causas externas. A relação de mortalidade entre os sexos passou de 2,1 em 1980 para 2,5 em 1995. ${ }^{1}$

Abrantes et al, 1998, estudaram as causas mais freqüentes de internações de crianças e adolescentes nos hospitais do Estado de Minas Gerais conveniados ao SUS, nos anos de 1994 e 1995, encontrando 9,5\% do total de internações na faixa etária da adolescência. Predominaram as internações para o sexo masculino (56,7\% dos 10 aos 14 anos), exceto para os indivíduos com idades entre os 15 e os 19 anos, faixa etária que em predominaram as internações relacionadas à gestação $(78,9 \%)$. As doenças respiratórias foram a principal causa de internação em todas as faixas etárias $(14,2 \%$ e 12,3\%, dos 10 aos 14 anos, respectivamente para os sexos masculino e feminino), excetuando as moças dos 15 aos 19 anos, seguida das doenças do trato digestório (7,9\% masculino e 2,3\% feminino, dos 10 aos 14 anos e 3,2\% masculino e $2 \%$ feminino, entre 15 e 19 anos). ${ }^{16}$

Embora no presente estudo as causas externas não apareçam como uma das principais causas de internação deve-se destacar que estas representam importante causa de morbidade e mortalidade entre os adolescentes. As causas externas interferem drasticamente na vida do adolescente, modificam e marcam caminhos, mutilam e subtraem anos potenciais de vida, deixando seqüelas físicas e emocionais e interferindo na dinâmica familiar. Estão relacionadas diretamente com a urbanização acelerada, migração interna, desigualdades sociais e marginalização, fatores indissociáveis da violência que acomete todas as faixas etárias, principalmente no sexo masculino, devido à maior exposição ao álcool, tabagismo, drogas, inserção no mercado de trabalho informal e atividades ilícitas. ${ }^{17,18}$

Em hospitais norte-americanos os acidentes foram identificados como a principal causa de internação entre os adolescentes de 10 a 14 anos de ambos os sexos e para os rapazes com mais de 15 anos, enquanto que as complicações da gravidez determi- naram a maioria das internações na faixa etária dos 15 aos 19 anos. ${ }^{19}$ Achados semelhantes também foram relatados no Brasil, sendo identificadas as causas externas como a principal causa de mortalidade entre adolescentes, principalmente no sexo masculino e na faixa etária dos 15 aos 19 anos. $^{20}$

$\mathrm{Na}$ Inglaterra, um estudo revelou predomínio de hospitalização entre os meninos com idades até 14 anos, cujas principais causas foram doenças do aparelho respiratório, traumatismos, apendicectomia e doenças das vias aéreas superiores, enquanto que para as meninas, as causas mais importantes foram adenoamigdalectomia, apendicectomia e traumatismos. Para a faixa etária dos 15 aos 19 anos, houve predomínio do sexo feminino, relacionado ao término da gravidez, enquanto que para os rapazes traumatismos e apendicectomia representaram as principais causas de internação. ${ }^{21}$ Siqueira e Tanaka, 1986, utilizando dados do Censo Demográfico Brasileiro de 1980, analisaram a mortalidade entre adolescentes de 10 a 19 anos e encontraram com principal diagnóstico as causas externas $(47,2 \%)$, seguido por doenças do aparelho circulatório $(6,8 \%)$, doenças infecciosas $(6,3 \%)$ e neoplasias $(5,9 \%)$. As complicações da gravidez, parto e puerpério foram responsáveis por $6,14 \%$ dos óbitos na faixa etária dos 15 aos 19 anos. $^{22}$

Alguns autores analisaram especificamente a mortalidade por causas externas, com o estudo de Barros et al 2001. ${ }^{17}$ Os resultados mostraram ser esta a primeira causa de mortalidade entre adolescentes, responsável por $65 \%$ dos óbitos no sexo masculino e $17,4 \%$ no feminino, tendo seus coeficientes aumentados gradativamente no período de 1979 a 1995. Martins e Andrade 2005, Londrina (PR), estudaram causas de atendimento hospitalar ou de morte em menores de 15 anos vítimas de causas externas, no ano de 2001. Dos 8854 casos atendidos em pronto-socorro, $34 \%$ foram devido a quedas, $19,5 \%$ a acidentes de trânsito e $13,5 \%$ de envenenamentos, ou seja, causas preveníveis. ${ }^{23}$

Outro aspecto que merece ser destacado devido ao potencial de risco para os adolescentes, mas com grandes possibilidades de êxito em atividades preventivas, são as questões relacionadas à sexualidade. A iniciação sexual ocorrendo no início da adolescência, o desconhecimento sobre saúde reprodutiva e a pouca utilização de métodos contraceptivos tornamse motivos de grande preocupação em virtude da ocorrência de gestações indesejáveis e disseminação de doenças sexualmente transmissíveis. A gravidez na adolescência é considerada um problema de saúde 
pública pelas muitas implicações para mãe, filho e toda a sociedade. Embora a incidência de gravidez e suas complicações estejam diminuindo, como reflexos de ações de saúde públicas voltadas especificamente aos adolescentes, ainda representam a maior causa de internação entre as moças com idades acima de 15 anos, reflexos da elevada fecundidade e da repetição da gravidez. $^{24,25}$

A morbidade por câncer também merece ser destacada visto que, segundo dados do Instituto Nacional do Câncer, as neoplasias, principalmente as Leucemias, os Linfomas e os Tumores do Sistema Nervoso Central, constituem a segunda causa de morte na faixa etária de 5 a 19 anos, ultrapassada apenas pelos óbitos por causas externas. ${ }^{26}$

O conhecimento do perfil de morbidade entre os adolescentes adquire grande relevância no sentido em que propicia subsídios para a adoção de ações preventivas, exequiíveis em nível primário, e a adequação das estruturas secundária e terciária, destinadas ao tratamento e reabilitação. Na primeira condição, podem-se destacar os programas de Puericultura, de Hebiatria e de Pré-Natal, incorporando às suas consultas de rotina orientações quanto à prevenção de acidentes e de comportamentos de risco, imunizações, educação sexual e apoio emocional. Quanto aos serviços de pronto atendimento e hospitalares, é necessário destinar espaços específicos e número de leitos suficientes para servir à população de adolescentes, além do treinamento e capacitação dos recursos humanos, objetivando acolher e orientar os adolescentes, em todos os níveis.

\section{Agradecimentos}

Aos funcionários do Centro de Processamento de Dados Hospitalares do Departamento de Medicina Social da Faculdade de Medicina de Ribeirão Preto da Universidade de São Paulo pela gentileza em colaborar na compilação dos dados.

\section{ABSTRACT}

Study model: epidemiological survey. Introduction: Adolescence is a phase of great biological and emotional transformation associated with the definition of one's social role, and it is important to learn about the most prevalent health problems during this age range in order to better direct actions and resources aiming at assistance and preventive programs.

Objectives: to determine the profile of hospital morbidity among adolescents from the Ribeirão Preto region (SP) and their temporal evolution over the years of 1988, 1997 and 2006.

Methods: survey of data of the Center for the Processing of Hospital Data of the Department of Social Medicine, Faculty of Medicine of Ribeirão Preto, University of São Paulo, regarding all admissions of adolescents to the 34 hospitals of the 26 municipalities of the Regional Health Division of Ribeirão Preto (SP), during the years of 1988, 1997 and 2006.

Results: the main diagnoses of 10-to-14-year-old adolescents admitted to the hospitals were pneumonia (first cause of hospitalization in 1988 and 1997), diarrhea and appendicitis during all years studied, whereas intoxications and neoplasias were diagnosed only in 1988. For adolescents older than 15 years, high rates of hospitalization were observed due to problems related to pregnancy, delivery and puerperium, although pneumonia, diarrhea and appendicitis were still important diagnoses. Hospitalization and death predominated among males during all years surveyed. Males always presented higher death rates than females and the proportions of deaths per number of hospitalizations indicated higher frequencies among 10-to-14-year-old adolescents.

Conclusions: knowing the profile of morbidity among adolescents favors the adoption of important feasible actions at the primary level that should be incorporated into Puericulture, Hebiatry and into Prenatal Care Programs at outpatient clinics.

Key words: Adolescent. Hospitalization. Morbidity. Mortality. 


\section{Referências Bibliográficas}

1. Orlandi MHF, Schor N. Adolescentes e jovens: magnitude da mortalidade em Maringá/PR. Ciênc Cuid Saúde. 2002; 1:16370.

2. Mulye TP, Park J, Nelson CD, Adams SH, Irwin Jr CE, Brindis $\mathrm{CD}$. Trends in adolescent and young adult health in the United States. J Adolesc Health. 2009; 45: 8-24.

3. Duarte-Salles T, Pasarin MI, Borrell C, Rodrigues-Sariz M, Rajmil L, Ferrer $\mathrm{M}$ et al. Social inequalities in health among adolescents in a large southern European city. J Epidemiol Community Health Online First, published on December 8 2009 as 10.1136/jech.2009.090100

4. IBGE. http://www.ibge.gov.br/ibgeteen/datas/saude/ jovensmaes.html. Acessado: 05/07/2010.

5. Nascimento EMR, Mota E, Costa MCN. Custos das internações de adolescentes em unidades da rede regional integrada ao SUS em Salvador, Bahia. Epidemiol Serv Saúde. 2003;12:137-45.

6. Guzman CR, Cano MAT. O adolescente hospitalizado. Disponível em http://www.fen.ufg.bg/revista/revista2_2/ ado_hosp.html. Acessado: 02/08/2010

7. Almeida IS, Rodrigues BMRD, Simões SMF. Desvelando o cotidiano do adolescente hospitalizado. Rev Bras Enferm. 2005;58:147-51.

8. Yunes J, Primo E. Características da mortalidade em adolescentes brasileiros das capitais das unidades federais. Rev Saúde Pública. 1983; 17: 263-78

9. Françoso LA. Mortalidade na adolescência: grave problema de saúde pública. Rev Paul Pediatr. 2005; 23:164.

10. Dubuc IF, Ferrari RAP. Adolescentes atendidos num serviço público de urgência e emergência: perfil de morbidade e mortalidade. Rev Eletrônica Enferm. 2006;8:250-8. disponível em http://www.fen.ufg.br/revista/revista8_2/ v8n2a10.htm. Acessado: 17/08/2010

11. Netto A, Gondin L, Robledo HH, Romero KT. Causas de morte em adolescentes no Município de Mogi das Cruzes no período de 2000 a 2004. Rev Paul Pediatr. 2005; 23:165-9.

12. Lyra SMK, Goldberg T, Iyda M. Mortalidade de adolescentes em área urbana da região sudeste do Brasil, 1984 -1993. Rev Saúde Pública. 1996; 30: 587-91.

13. Roncevic N, Popadic-Gacesa J, Grujic V, Arsic M, Pericin I. Hospital morbidity and mortality of adolescents in Vojvodina. Med Pregl. 2009; 62:137-41.
14. Bochner R. Perfil das intoxicações em adolescentes no Brasil no período de 1999 a 2001. Cad Saúde Pública. 2006; 22: 587-95.

15. Nascimento EMR, Mota E, Costa MCN. Custos das internações de adolescentes em unidades da rede hospitalar integrada ao SUS em Salvador, Bahia. Epidemiol Serv Saúde. 2003;12:137-45

16. Abrantes MM, Lamounier JA, Faria JF, Diniz CM, Cunha FAF. Causas de internações de crianças e adolescentes nos hospitais do SUS em Minas Gerais entre 1994 e 1995. Inf Epidemiol SUS. 1998; 7: 95-104.

17. Barros MDA, Ximenes R, Lima MLC. Mortalidade por causas externas em crianças e adolescentes: tendências de 1979 a 1995. Rev Saúde Pública. 2001;35:142-9.

18. León JC, Carmona J, Garcia P. Health-risk behaviors in adolescents as indicators of unconventional lifestyles. J Adolesc. 2009; dois:10.1016/j.adolescence.2009.11.004

19. McManus M, McCarthy E, Kozak LJ, Newarcheck P. Hospital use by adolescents and young adults. J Adolesc Health. 1991;12:107-15

20. Lolio CA, Santo AH, Buchalla CM. Mortalidade de adolescentes no Brasil, 1977, 1980 e 1985. Magnitude e tendências. Rev Saúde Pública. 1990; 24: 481-9.

21. Henderson J, Goldacre M, Yeates D. Use of hospital inpatient care in adolescence. Arch Dis Child. 1993; 69: 559-63.

22. Siqueira AAF, Tanaka ACD. Mortalidade na adolescência com especial referência à mortalidade materna, Brasil, 1980. Rev Saúde Pública. 1986; 20: 274-9.

23. Martins CBG, Andrade SM. Causas externas entre menores de 15 anos em cidade do sul do Brasil: atendimentos em pronto-socorro, internações e óbitos. Rev Bras Epidemiol. 2005; 8: 194-204.

24. Yazlle MEHD, Franco RC, Michelazzo D. Gravidez na adolescência: uma proposta para prevenção. Rev Bras Ginecol Obstet. 2009;31:477-9

25. Bruno ZV, Feitosa FEL, Silveira KP, Morais IQ, Bezerra MF Reincidência de gravidez em adolescentes. Rev Bras Ginecol Obstet. 2009; 31: 480-4

26. Ministério da Saúde. Instituto Nacional do Câncer. Diagnóstico Precoce do Câncer na Criança e no Adolescente. Brasília. 2009. 114 p. 